\title{
PMC-Based Waveguide-Fed Slot Array
}

\section{Amir Jafargholi, Manouchehr Kamyab, and Mehdi Veysi}

\author{
E. E. Department, K. N. Toosi University of Technology, 19697 Tehran, Iran \\ Correspondence should be addressed to Amir Jafargholi, jafargholi@ee.kntu.ac.ir
}

Received 11 August 2010; Accepted 8 September 2010

Academic Editors: D. Liu, C. Luxey and G. Tsoulos

Copyright ( 12011 Amir Jafargholi et al. This is an open access article distributed under the Creative Commons Attribution License, which permits unrestricted use, distribution, and reproduction in any medium, provided the original work is properly cited.

Perfect magnetic conductors (PMCs) can help in the enhancement of antenna impedance bandwidth using their capability of reflecting the incident waves without phase reversal. The purpose of this paper is to show the advantages of using a perfect magnetic conductor in antenna engineering. The goal is to use it in waveguide-fed slot array antennas, increasing both the antenna impedance and radiation bandwidths. To this aim, a PMC-based rectangular waveguide composed of longitudinal slots is convenient. The impedance of the proposed array structure is calculated analytically. To compare analytical results with the simulation results, PEC- and PMC-based waveguide-fed slot arrays are designed and simulated in a certain frequency band. The simulation results are in good agreement with the theoretical predictions.

\section{Introduction}

Waveguide-fed slot arrays have been widely used in many spacecraft and radar applications due to the advantages of high efficiency, low weight, low profile, ease of manufacture, and rigid structure [1-3]. One of the main drawbacks of a waveguide-fed slot array is its narrow bandwidth, which is mainly caused by the use of resonant elements and the standing wave-type design.

Introducing metamaterials based on theory of negative index materials opened the way for many researcher groups all over the world to improve the operational performances of the electromagnetic-based devices [4-7]. One of the first works was done by Veselago in 1968 [4]. Recently, a typical procedure has been established for the design of bulk artificial media with negative macroscopic parameters. In recent years, several researcher groups have focused on metamaterial realization of the artificial magnetic conductors (AMCs) [8-11]. It was demonstrated in $[12,13]$ that artificial magnetic conductor can be realized using a volumetric metamaterial constructed from a periodic arrangement of the capacitively loaded loop (CLL) elements. Hence, it provides a very large number of applications in antenna engineering. Although a perfect magnetic conductor (PMC) does not exist in nature, the possibility to create artificial magnetic conductor (AMC) using engineered structures is promising [14].

In this paper, a novel application of perfect magnetic conductor is presented. A PMC-based waveguide-fed slot array, which provides broad bandwidth as well as endfire radiation, has been investigated both analytically and numerically. Consequently, typical PEC- and PMC-based waveguide-fed slot arrays have been designed and simulated. Results show that the agreement between the numerical simulations and theoretical predictions is reasonably good.

\section{Design of PMC-Based Waveguide-Fed Slot Array}

The simulated model of a PMC-based waveguide-fed slot array designed to work at $f=2.5 \mathrm{GHz}$ is shown in Figure 1 . The feed port is located at input of the air-filled waveguide of rectangular cross-section while the end is shorted. According to the theory presented in [15-17], the radiation characteristics of a slot on infinite PMC plate arederived 
as

$$
\begin{gathered}
H_{r}(\theta, \phi) \approx 0, \\
H_{\theta}(\theta, \phi)=j \frac{w L k H_{0} e^{-j k r}}{2 \pi r}\left(\sin \phi \frac{\sin (X)}{X} \frac{\sin (Y)}{Y}\right), \\
H_{\phi}(\theta, \phi)=j \frac{w L k H_{0} e^{-j k r}}{2 \pi r}\left(\cos \theta \cos \phi \frac{\sin (X)}{X} \frac{\sin (Y)}{Y}\right), \\
E_{r}(\theta, \phi) \approx 0, \\
E_{\theta}(\theta, \phi)=j \eta \frac{w L k H_{0} e^{-j k r}}{2 \pi r}\left(\cos \theta \cos \phi \frac{\sin (X)}{X} \frac{\sin (Y)}{Y}\right), \\
E_{\phi}(\theta, \phi)=-j \eta \frac{w L k H_{0} e^{-j k r}}{2 \pi r}\left(\sin \phi \frac{\left.\sin (X) \frac{\sin (Y)}{X}\right),}{Y}\right)
\end{gathered}
$$

where

$$
X=\frac{k w}{2} \sin \theta \cos \phi, \quad Y=\frac{k L}{2} \sin \theta \sin \phi
$$

where $w$ and $L$ are the width and length of the slot, respectively. The waveguide dimensions are $a=8.636 \mathrm{~cm}$ and $b=4.318 \mathrm{~cm}$ (WR340). The distance between the last slot and the end of the waveguide is $9.906 \mathrm{~cm}$ while the distance between the first slot and the waveguide port is about $15 \mathrm{~cm}$. It is worthwhile to point out that our approach is mainly based on theory presented in [17]. One can now calculate the antenna radiation conductance as follows:

$$
\begin{aligned}
& W_{a v}=\frac{1}{2} \operatorname{Re}\left(E \times H^{*}\right) \\
& =\frac{1}{2} \operatorname{Re}\left(a_{\theta} E_{\theta} \times a_{\phi} H_{\phi}^{*}+a_{\phi} E_{\phi} \times a_{\theta} H_{\theta}^{*}\right) \\
& =\frac{\eta}{2}\left\{\left[\frac{w L k H_{0}}{2 \pi r}\left(\frac{\sin (X)}{X} \frac{\sin (Y)}{Y}\right)\right]^{2}\right. \\
& \left.\cdot\left[(\cos \theta \cos \varphi)^{2}+(\sin \varphi)^{2}\right]\right\}, \\
& P_{\mathrm{rad}}=\oiiint_{s} W_{a v} \cdot d s=\frac{\eta}{2} \oiint_{s} a_{r} W_{a v} \cdot a_{r} r^{2} \sin \theta d \theta d \varphi \\
& =\frac{\eta}{2}\left(\frac{w L k H_{0}}{2 \pi}\right)^{2}
\end{aligned}
$$

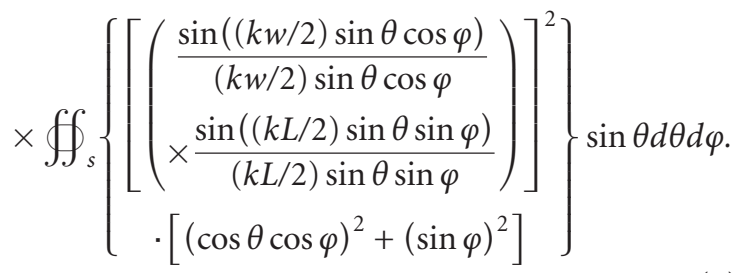

Using Duality theorem, one can readily deduce that

$$
V_{0}=w E_{0} \stackrel{\text { Duality }}{\Longrightarrow}-H_{0}=\frac{I_{0}}{w} .
$$

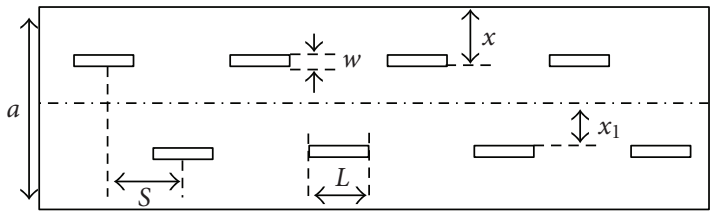

(a)

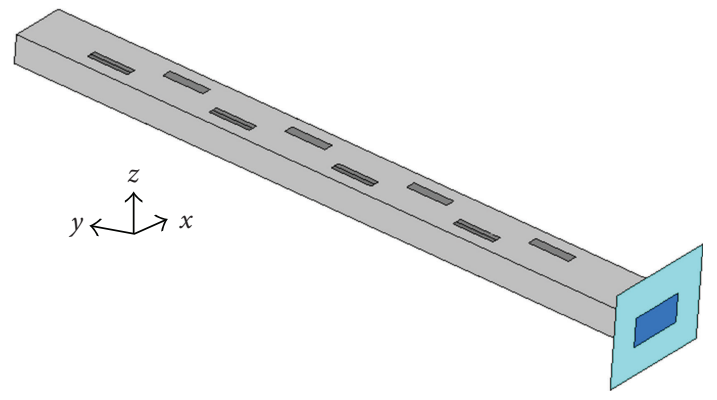

(b)

Figure 1: A schematic of PMC-based waveguide-fed slot array antenna: $L=6.5024 \mathrm{~cm}, S=9.906 \mathrm{~cm}, w=1.5 \mathrm{~cm}, x=3.568 \mathrm{~cm}$, and $x_{1}=2.068 \mathrm{~cm}$.

Insertion of (5) in (4) gives

$$
\begin{aligned}
& P_{\mathrm{rad}} \\
& =\frac{1}{2} R_{\mathrm{rad}} I_{0}^{2} \Longrightarrow R_{\mathrm{rad}} \\
& =\frac{4 \eta}{(k \pi w)^{2}} \\
& \times \int_{0}^{\pi} \int_{0}^{2 \pi}\left\{\left[\frac{\left.\left(\frac{\sin \left(\frac{k w}{2} \sin \theta \cos \varphi\right) \cdot \sin \left(\frac{k L}{2} \sin \theta \sin \varphi\right)}{\sin ^{3} \theta \cos ^{2} \varphi \sin ^{2} \varphi}\right)\right]}{\left.\times\left[(\cos \theta \cos \varphi)^{2}+(\sin \varphi)^{2}\right]\right\} d \theta d \phi,}\right.\right.
\end{aligned}
$$

Expanding the following terms using Taylor expansion gives

$$
\begin{aligned}
& \sin \left(\frac{k w}{2} \sin \theta \cos \phi\right) \approx \frac{k w}{2} \sin \theta \cos \phi-\frac{((k w / 2) \sin \theta \cos \phi)}{3 !} \\
& \sin \left(\frac{k L}{2} \sin \theta \sin \phi\right) \approx \frac{k L}{2} \sin \theta \sin \phi-\frac{((k L / 2) \sin \theta \sin \phi)^{3}}{3 !}
\end{aligned}
$$

Finally, some mathematical manipulations and singularity cancelations give

$$
R_{\mathrm{rad}}=\eta \frac{L}{6 w \pi} k^{2} \cdot\left(L^{2}-\frac{w^{2}}{3}\right) .
$$


The antenna radiation power then becomes

$$
P_{\text {rad }}=\frac{1}{2} R_{\mathrm{rad}} I_{0}^{2}=\eta \frac{L}{12 w \pi} k^{2} \cdot\left(L^{2}-\frac{w^{2}}{3}\right) I_{0}^{2} .
$$

For the PMC-based waveguide-fed slot array of Figure 1, the antenna radiation power can be also written as [17]

$$
P_{\mathrm{rad}}=\frac{\omega \varepsilon_{0} a b \beta_{10}}{2(\pi / a)^{2}} B_{10}\left(A_{10}+B_{10}\right),
$$

where $A_{10}$ is the amplitude of $T E_{10}$ mode launched by the generator and $B_{10}$ is the amplitude of $T E_{10}$ mode scattered off the slot. Then, using the same procedure presented in [17], the normalized slot resistance can be written in the form

$$
\begin{aligned}
r= & \frac{R_{\mathrm{rad}}}{R_{\mathrm{rad}, 0}}=\frac{2 B_{10}}{\left(A_{10}+B_{10}\right)} \\
= & \frac{24}{\pi k^{2}} \cdot \frac{w}{L\left(L^{2}-w^{2} / 3\right)} \\
& \cdot \frac{(a / b)}{\left(\beta_{10} / k\right)}\left(\cos \beta_{10} l-\cos k l\right)^{2} \cos ^{2} \frac{\pi x}{a},
\end{aligned}
$$

where $R_{10}$ is the characteristic resistance of the feeding waveguide and $l=L / 2$. For the example discussed here, $x=a / 2-x_{1}, k l=\pi / 2$. So that (11) then becomes

$$
\begin{aligned}
r= & \frac{R_{\mathrm{rad}}}{R_{\mathrm{rad}, 0}} \approx \frac{24}{\pi k^{2}} \cdot \frac{w}{L\left(L^{2}-w^{2} / 3\right)} \\
& \cdot \frac{(a / b)}{\left(\beta_{10} / k\right)} \cos ^{2}\left(\frac{\pi \beta_{10}}{2 k}\right)^{2} \sin ^{2} \frac{\pi x_{1}}{a} .
\end{aligned}
$$

Hence,

$$
r_{\mathrm{PMC}}=\frac{24}{\pi k^{2}} \cdot \frac{w}{L\left(L^{2}-w^{2} / 3\right)} \cdot K(f),
$$

where

$$
K(f)=\frac{(a / b)}{\left(\beta_{10} / k\right)} \cos ^{2}\left(\frac{\pi \beta_{10}}{2 k}\right) \sin ^{2} \frac{\pi x}{a},
$$

whereas the normalized slot conductance associated with PEC-based waveguide-fed slot array is given by [17]

$$
g_{\text {PEC }}=2.09 K(f) .
$$

As a result, the variation in the normalized slot resistance as a function of frequency for the PMC-based waveguide-fed slot array becomes smaller than that for the PEC case. Thus, one expects that PMC-based waveguide-fed slot array has wider bandwidth compared to the PEC-based waveguide-fed slot array. Comparing (13) to (15), one can conclude that the variation of the radiation conductance for PEC-based rectangular-fed slot array is about 14 times greater compared to the radiation resistance of the PMC case (at around $f=2.5 \mathrm{GHz}$ ). In other words, the PMC-based rectangularfed slot array bandwidth can be determined by multiplying PEC-based rectangular-fed slot array bandwidth by 14 . In addition, the main lobe of the PEC-based waveguide-fed slot array is in the broadside direction. In contrast, the main lobe of the PMC-based waveguide-fed slot array is in the end-fire direction.

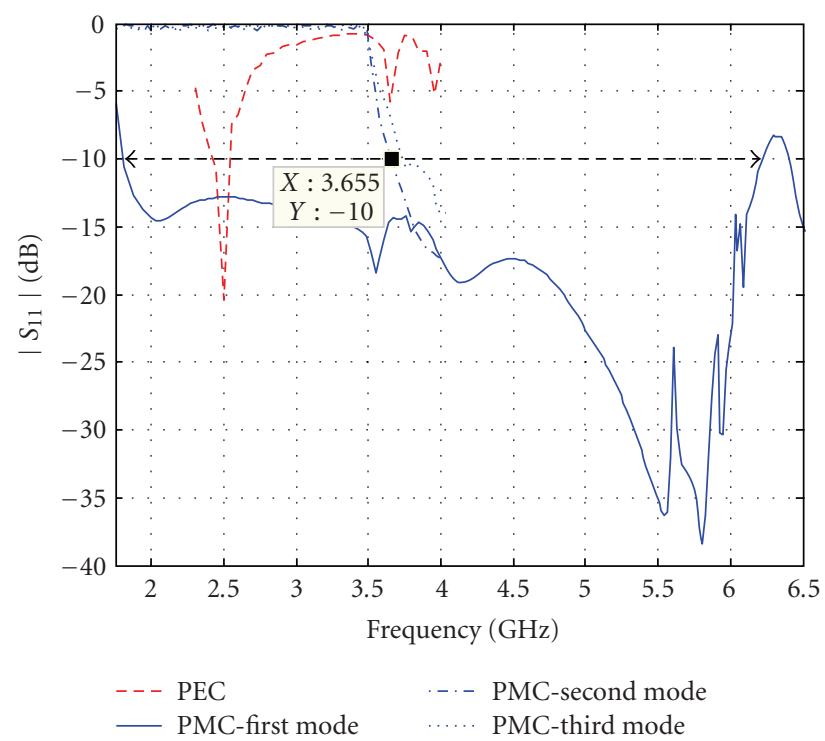

Figure 2: Return loss of the PMC- and PEC-based waveguide-fed slot arrays.

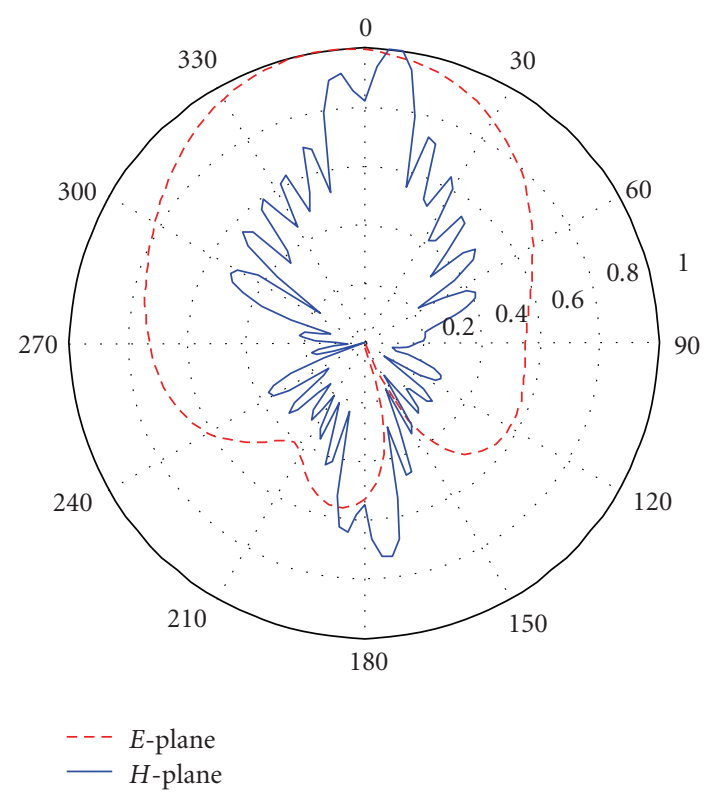

FIgURE 3: Normalized radiation pattern of the PEC-based waveguide-fed slot array at $f_{0}=2.5 \mathrm{GHz}$.

\section{Simulation Results}

To verify the proposed antenna concept, PMC- and PECbased waveguide-fed slot arrays were designed and simulated. The simulated return loss of both antennas is plotted in Figure 2. As revealed in the figure, bandwidth of the PEC-based waveguide-fed slot array is approximately $4.8 \%(0.12 \mathrm{GHz})$. In contrast to ordinary waveguide, the bandwidth of the PMC-based waveguide-fed slot array is approximately $110 \%(4.4 \mathrm{GHz}$, ranging from $1.8 \mathrm{GHz}$ to $6.2 \mathrm{GHz}$ ). It can be concluded from the simulation results that the bandwidth of the PMC-based 


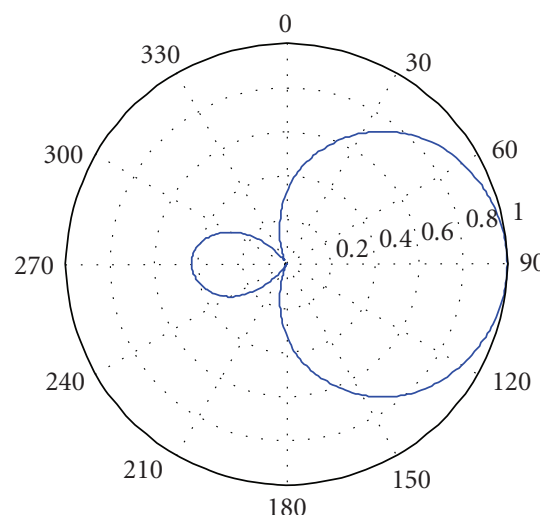

$-2 \mathrm{GHz}$

(a)

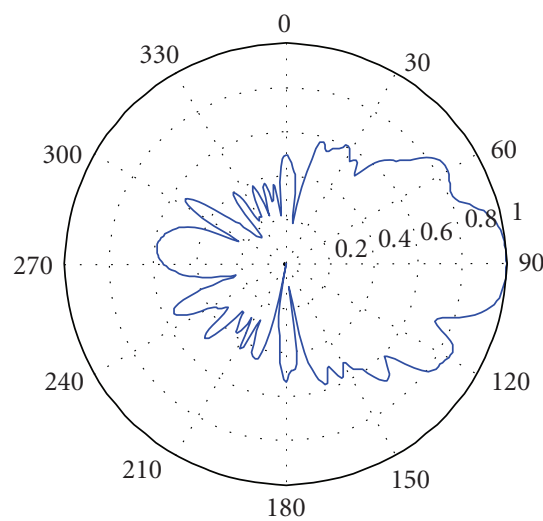

$-3.5 \mathrm{GHz}$

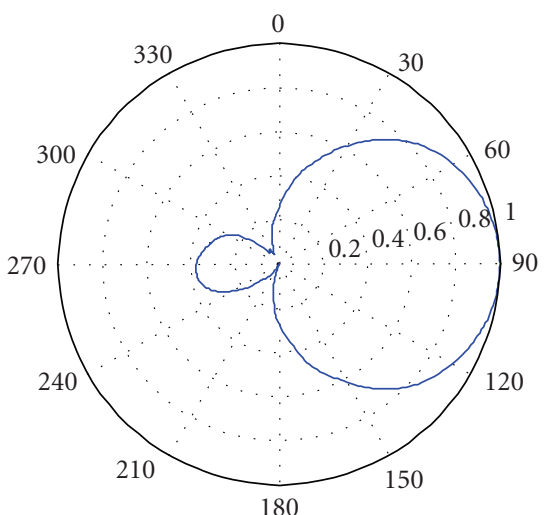

$-2.5 \mathrm{GHz}$

(b)

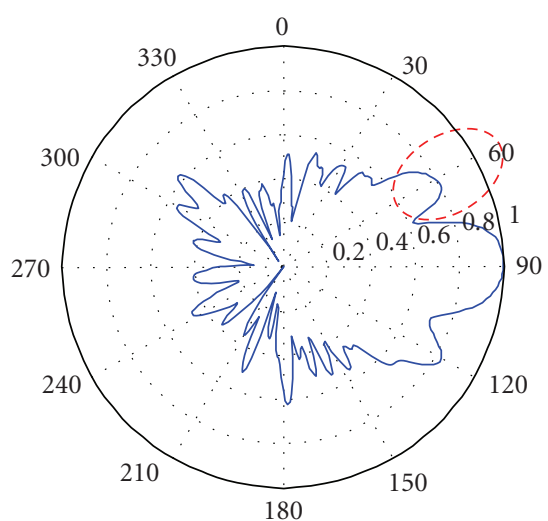

$-3.7 \mathrm{GHz}$

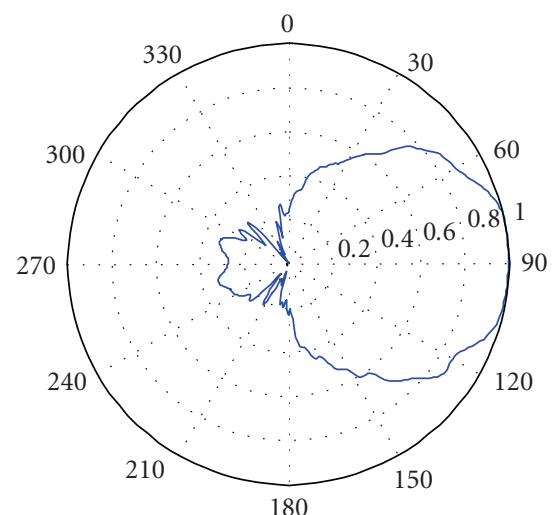

$-3 \mathrm{GHz}$

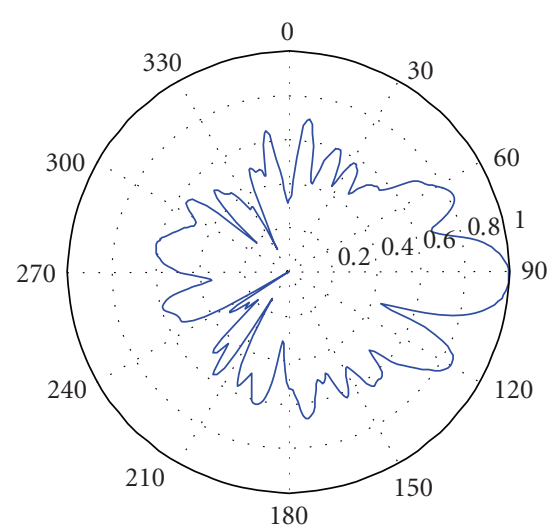

$-3.8 \mathrm{GHz}$

(e)

(f)

FIGURE 4: CST simulation results: the normalized radiation patterns of the PMC-based waveguide-fed slot array in E-plane and at (a) 2 GHz, (b) $2.5 \mathrm{GHz}$, (c) $3 \mathrm{GHz}$, (d) $3.5 \mathrm{GHz}$, (e) $3.7 \mathrm{GHz}$, and (f) $3.8 \mathrm{GHz}$.

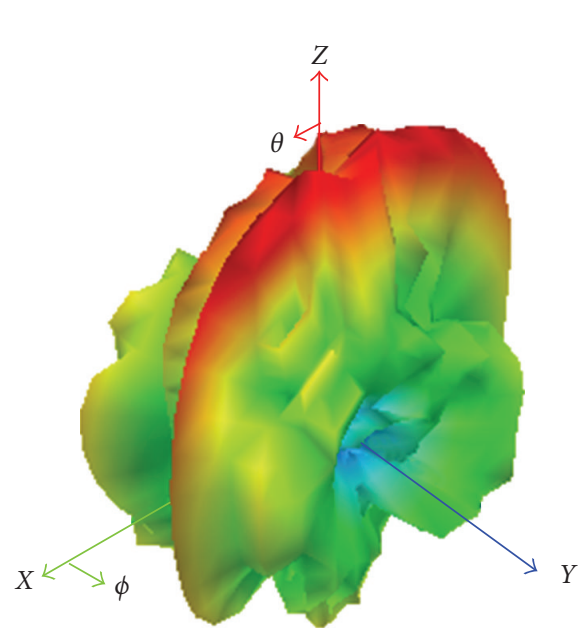

(a)

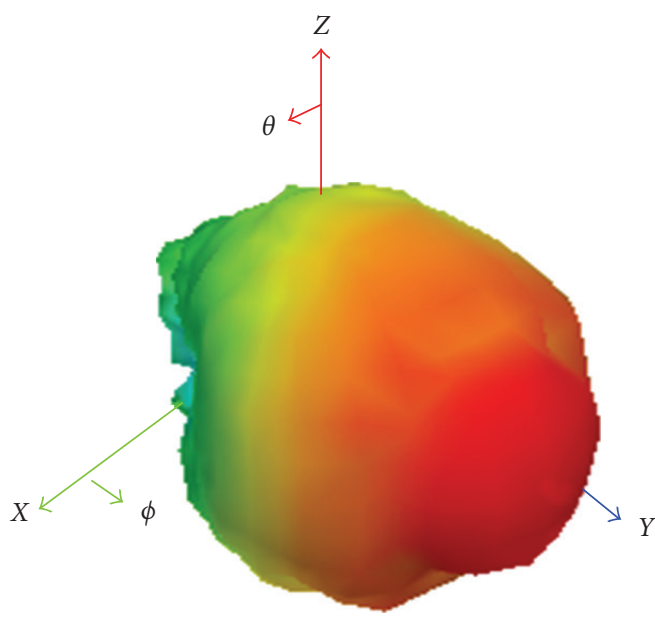

(b)
dB (gain total) $1.2969 e+001$ $1.0255 e+001$ $7.5404 e+000$ $4.826 e+000$ $2.1116 e+000$ $-6.0279 e-001$ $-3.3172 e+000$ $-6.0316 e+000$ $-8.746 e+000$

$-1.146 e+001$

$-1.4175 e+001$ $-1.6889 e+001$ $-1.9604 e+001$ $-2.2318 e+001$

$-2.7747 e+001$
$7.4924 e+000$ $6.0414 e+000$ $4.5904 e+000$

$3.1394 e+000$

$1.6884 e+000$

$2.3739 e-001$

$-1.2136 e+000$

$-2.6646 e+000$

$-4.1156 e+000$

$-5.5666 e+000$

$-7.0176 e+000$

$-8.4686 e+000$

$-9.9196 e+000$

$-1.1371 e+001$

$-1.2822 e+001$

$-1.4273 e+001$

$-1.5724 e+001$

FIgURe 5: 3D radiation pattern for 8-element (a) PEC- and (b) PMC-based waveguide-fed slot arrays at $f=2.5 \mathrm{GHz}$. 
rectangular-fed slot array is about $4.4 / 0.12 \approx 36.7$ times greater compared to that of the PEC-based rectangular-fed slot array. Although disagreement between the numerical and theoretical results is observed, more careful investigations clarify that. The reason is that for wideband operation, both the return loss and radiation pattern requirements should be satisfied in the entire frequency band.

In other words, the operational frequency band is the common portion of the impedance and radiation bandwidth. Therefore, radiation patterns of the PMCbased waveguide-fed slot array should be extracted and compared with those of the PEC-based waveguide-fed slot array over the entire frequency band. Figure 3 shows the radiation pattern of the PEC-based waveguide-fed slot array at $2.5 \mathrm{GHz}$. As revealed in the figure, the array has a fan beam and the main lobe direction is perpendicular to the array plane.

Figure 4 shows the radiation patterns of the PMC-based waveguide-fed slot array at six different frequencies. As can be seen, the maximum directivity occurs at $\theta=90^{\circ}$ whereas the radiation bandwidth is around 61\% (ranging from $1.95 \mathrm{GHz}$ to $3.65 \mathrm{GHz}$ ). At frequencies beyond $3.6 \mathrm{GHz}$, antenna radiation pattern has been distorted. The likely cause is that the higher-order modes start to appear at frequency higher than $3.6 \mathrm{GHz}$ (see Figure 2). The presence of the undesired modes causes not only higher losses but also radiation pattern distortion. In other words, one can observe significant null in the antenna main lobe at frequencies beyond $3.65 \mathrm{GHz}$, as shown in Figure 4. And consequently, numerical investigation reveals that the bandwidth of the PMC-based rectangular-fed slot array is about 14.16 times greater compared to that of the PEC-based rectangular-fed slot array, which is in good agreement with the theoretical predictions. The $3 \mathrm{D}$ radiation patterns of the PEC- and PMC-based waveguide-fed slot arrays are compared in Figure 5. As can be seen, the gain of the PMC-based slot array is approximately half of that of the PEC case, which is the main disadvantage associated with this antenna. In addition the slot array does not have a fan beam any more. Finally, it should be pointed out that the gain of the slot array decreases with frequency which is partly due to the resonant nature of the array elements and partly due to the variations of the element separation with frequency. In other words, Very high radiation and impedance bandwidths can be achieved by an end fire radiation pattern with low minor lobes at the expense of a decrease of about $7 \mathrm{~dB}$ in gain.

\section{Conclusion}

A new PMC-based rectangular waveguide-fed slot array has been investigated which provides UWB radiation bandwidth as well as end-fire radiation. Radiation conductance of the proposed array has been calculated analytically. PEC- and PMC-based waveguide-fed slot arrays have been designed and simulated to validate and confirm the theoretical predictions. The simulated results are in good agreement with the theoretical calculations.

\section{References}

[1] H. Y. Yee, "The design of large waveguide arrays of shunt slots," IEEE Transactions on Antennas and Propagation, vol. 40, no. 7, pp. 775-781, 1992.

[2] J. C. Coetzee, J. Joubert, and W. L. Tan, "Frequency performance enhancement of resonant slotted waveguide arrays through the use of wideband radiators or subarraying," Microwave and Optical Technology Letters, vol. 22, no. 1, pp. 35-39, 1999.

[3] R. S. Elliott, "On the design of traveling-wave-fed longitudinal shunt slot arrays," IEEE Transactions on Antennas and Propagation, vol. 27, no. 5, pp. 717-720, 1979.

[4] V. G. Veselago, "The electrodynamics of substances with simultaneously negative values of $\varepsilon$ and $\mu$," Soviet Physics Uspeekhi, vol. 10, pp. 509-514, 1968.

[5] D. R. Smith, W. J. Padilla, D. C. Vier, S. C. Nemat-Nasser, and S. Schultz, "Composite medium with simultaneously negative permeability and permittivity," Physical Review Letters, vol. 84, no. 18, pp. 4184-4187, 2000.

[6] R. A. Shelby, D. R. Smith, and S. Schultz, "Experimental verification of a negative index of refraction," Science, vol. 292, no. 5514, pp. 77-79, 2001.

[7] C. Caloz, C.-C. Chang, and T. Itoh, "Full-wave verification of the fundamental properties of left-handed materials in waveguide configurations," Journal of Applied Physics, vol. 90, no. 11, pp. 5483-5486, 2001.

[8] D. Sievenpiper, L. Zhang, R. F. Jimenez Broas, N. G. Alexöpolous, and E. Yablonovitch, "High-impedance electromagnetic surfaces with a forbidden frequency band," IEEE Transactions on Microwave Theory and Techniques, vol. 47, no. 11, pp. 2059-2074, 1999.

[9] R. Coccioli, F.-R. Yang, K.-P. Ma, and T. Itoh, "Aperturecoupled patch antenna on uc-pbg substrate," IEEE Transactions on Microwave Theory and Techniques, vol. 47, no. 11, pp. 2123-2130, 1999.

[10] D. Sievenpiper, H.-P. Hsu, J. Schaffner, G. Tangonan, R. Garcia, and S. Ontiveros, "Low-profile, four-sector diversity antenna on high-impedance ground plane," Electronics Letters, vol. 36, no. 16, pp. 1343-1345, 2000.

[11] J. McVay, N. Engheta, and A. Hoorfar, "High-impedance metamaterial surfaces using Hilbert-curve inclusions," IEEE Microwave and Wireless Components Letters, vol. 14, no. 3, pp. 130-132, 2004.

[12] R. W. Ziolkowski, "Design, fabrication, and testing of double negative metamaterials," IEEE Transactions on Antennas and Propagation, vol. 51, no. 7, pp. 1516-1529, 2003.

[13] A. Erentok, P. L. Luljak, and R. W. Ziolkowski, "Antenna performance near a volumetric metamaterial realization of an artificial magnetic conductor," IEEE Transactions on Antennas and Propagation, vol. 53, no. 1, pp. 160-172, 2005.

[14] N. Engheta and R. W. Ziolkowski, Metamaterials Physics and Engineering Explorations, Wiley-Interscience, New York, NY, USA, 2006.

[15] J. Volakis, Antenna Engineering Handbook, McGraw-Hill, New York, NY, USA, 4th edition, 2007.

[16] C. A. Balanis, Antenna Theory: Analysis and Design, John Wiley \& Sons, New York, NY, USA, 2003.

[17] R. S. Elliott, Antenna Theory and Design, John Wiley \& Sons, New York, NY, USA, 2003. 

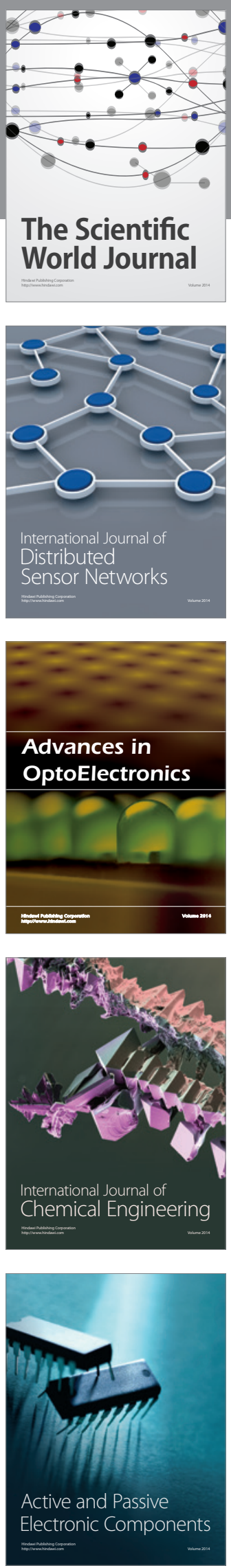
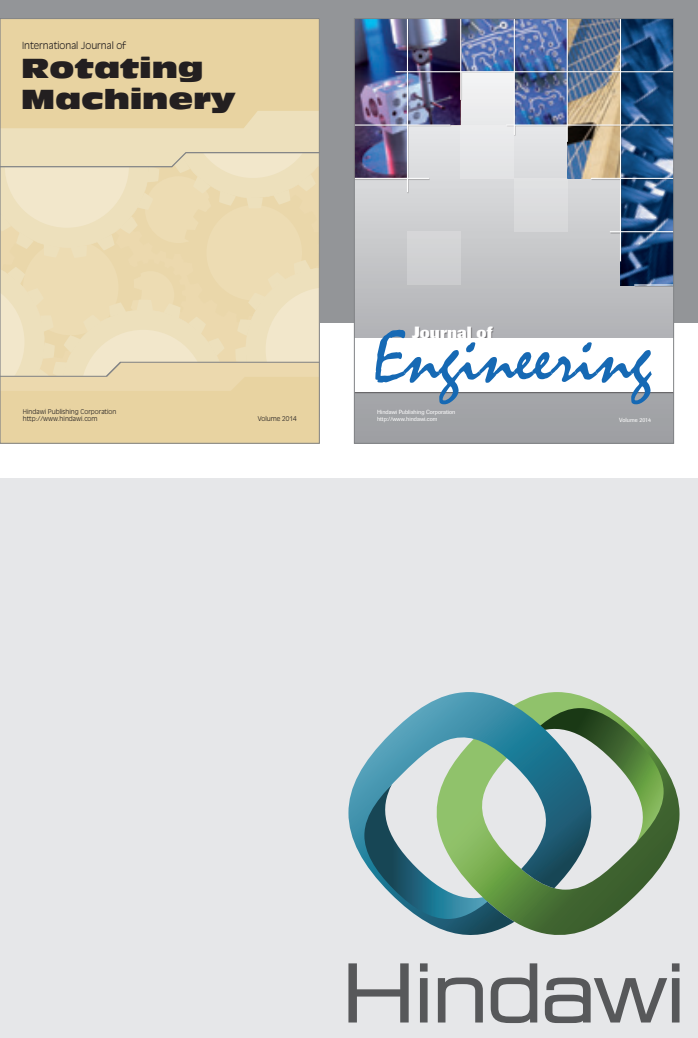

Submit your manuscripts at

http://www.hindawi.com
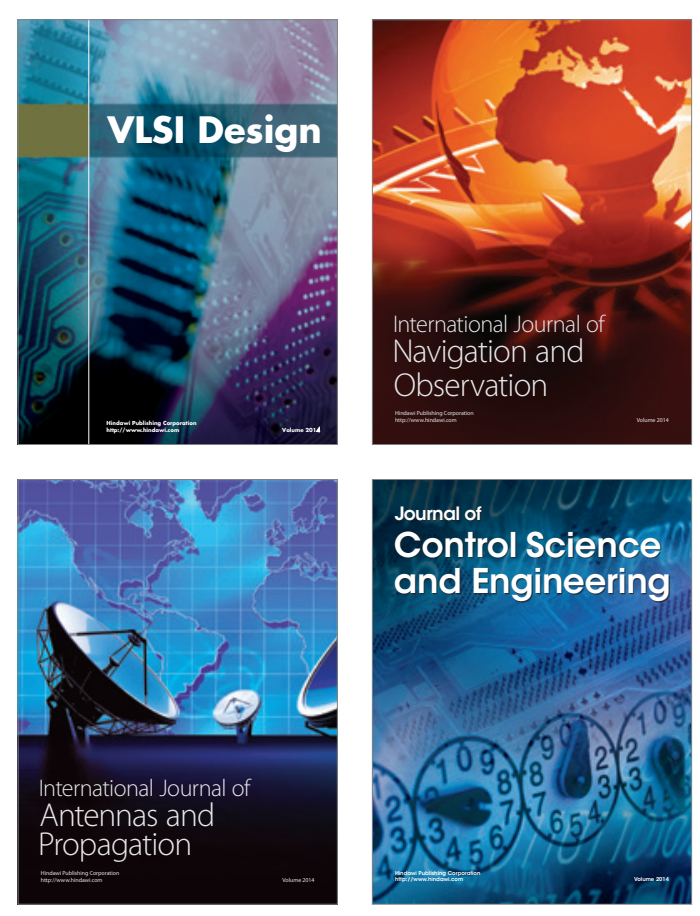
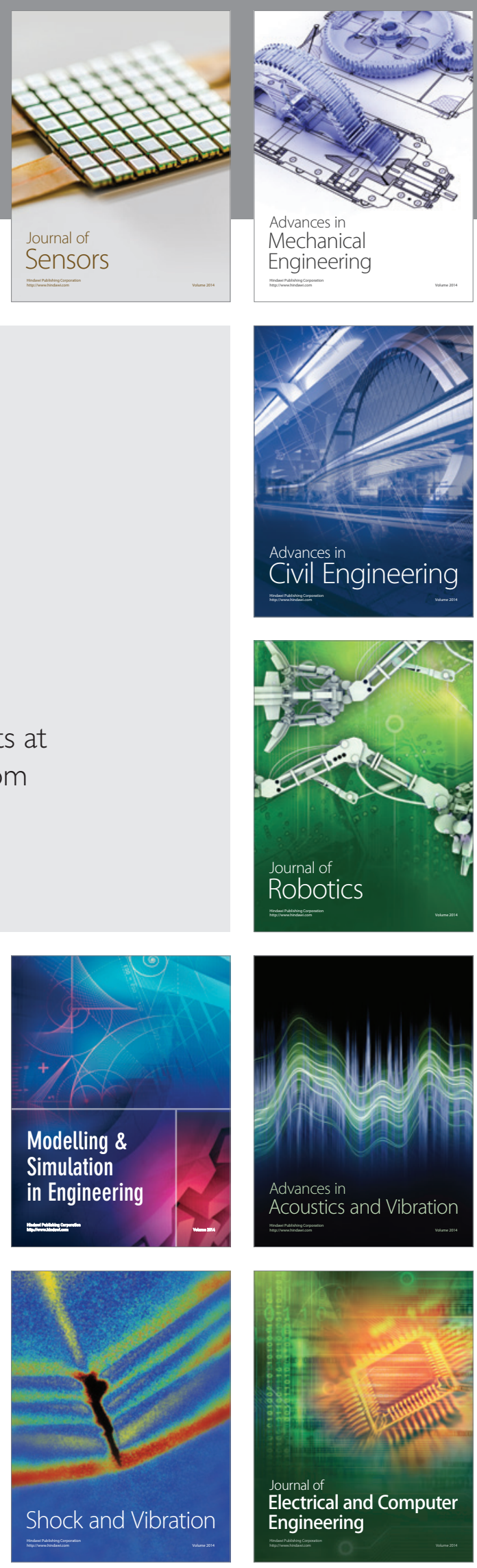